\title{
Torsional evaluation of Tapered Composite Cone using Finite Element Analysis
}

\author{
Onkar Pawar $^{1}$, R.M.Tayade ${ }^{2}$,V.G.Patil ${ }^{3}$, Manan Panchal ${ }^{4}$ \\ ${ }^{1,2}$ (Mechanical Dept., V.J.T.I Mumbai, India) \\ ${ }^{3,4}$ (FEA Consultant, Vaftsy CAE Institute, India)
}

\begin{abstract}
Composite material is one of the most important and economical material for the various application due to its favorable properties. Recently many researches are going on the various properties of the these materials. In this paper an anisotropic behavior of the composite tube is to be modeled and analysis is to be performed under torsional loading conditions. Torsion is a tricky phenomenon in composite cylinders as the twist effects and their interactions with composite shells induce complex stress patterns. The objective behind the study is to understand interaction of conical angle, length of tube and torsional moment it also includes comparative analysis of deformation and stresses developed in tapered composite cone due to use of various materials like steel, orthotropic composite and laminated composite etc. The effect of taper angle, thickness of the tube and fiber orientations in case of laminated composite is studied by using finite element analysis (ANSYS software). The finite element analysis is especially versatile and efficient for the analysis of complex structural behavior of the composite laminated structures. It is found that deformation in case of laminated composite and deformation in between steel and laminated composite cone. At membrane stresses are observed at the middle of cone in length direction for three materials.
\end{abstract}

Keywords: Torsional analysis, Laminated Composites, Finite Element Analysis

Accepted Date: 24 June 2013

\section{Introduction}

An application of composite materials in automobile, aeronautical and other engineering structures are ever increasing, due to their highly desirable properties like high specific strength/ stiffness, low co-efficient of expansion, damping properties and directional dependence.

Composite shells are increasing being used in Cone Structures for various applications across the board. Typical applications are in sports equipment such as golf sticks, tennis rackets, compressed natural gas (CNG) tanks etc. In automobile, high speed vehicles use conical tubes in diffuser arrangement. Other applications of composite shells include wind mill blades, rollers, shafts and X ray inspection equipment. Laminated cylindrical shells are used in pressure vessels, missiles and aircraft, fuselage structures etc. Laminated conical shells are often used as transition elements between cylinders of different diameters, end closures etc. The use of laminated composites in conical shells are however limited to tubes of low radius ratio, since high radius ratios results in a non-uniform orientation of fibers in a ply.

Laminated composite shells in particular are prominent in bearing various types of loads and are hence used in many engineering structures. The analysis of laminated composites is quite complicated since the material behavior is anisotropic, which gets further intensified in the analysis of complex structures like shells. In materials science, Composite laminates are assemblies of layers of fibrous composite materials which can be joined to provide required engineering properties, including in-plane stiffness, bending stiffness, strength, and coefficient of thermal expansion. The individual layers consist of high-modulus, high-strength fibers in a polymeric, metallic, or ceramic matrix material. Typical fibers used include graphite, glass, boron, and silicon carbide, and some matrix materials are epoxies, polyimide, aluminum, titanium, and alumina. Layers of different materials may be used, resulting in a hybrid laminate. The individual layers generally are orthotropic (that is, with principal properties in orthogonal directions) or transversely isotropic (with isotropic properties in the transverse plane) with the laminate then exhibiting anisotropic (with variable direction of principal properties), orthotropic, or quasi-isotropic properties. Quasi-isotropic laminates exhibit isotropic (that is, independent of direction) in plane response but are not restricted to isotropic out-of-plane (bending) response. Depending upon the stacking sequence of the individual layers, the laminate may exhibit coupling between in plane and out-of-plane response. An example of bending-stretching coupling is the presence of curvature developing as a result of in-plane loading.

In the past, the structural behavior of plates and shells using the finite element method has been studied by a variety of approaches. Chethana Shankara Rao (2007)[1] analyzed tapered composite tubes under torsion and 
tension .they studied the effect The effect of stacking sequence, taper angle and fiber orientations on the axial deformation and twisting angle are by using the developed method. Brian Schmalberger (May 2010)[2] work on Optimization of an Orthotropic Composite Beam. In this they analyzed the response of an orthotropic composite beam as the fiber orientation angle in the lamina increases from $0^{\circ}$ to $90^{\circ}$. Specifically, the response to a point load and the modes of vibration are simulated. Choudhary and Tungikar (2011) [3] analyzed the geometrically nonlinear behavior of laminated composite plates using the finite element analysis. They studied the effect of number of layers, effect of degree of orthotropy (both symmetric and ant symmetric) and different fibre orientations on central deflections.

In this paper, we are analyzing torsional behavior of tapered cone with different material and different thickness values of a cone. In this, three types of materials namely steel, orthotropic composite and laminated composite (with 0/45/90 ply arrangement) are used for analysis of tapered composite cone. In laminated composite material $(0 / 45 / 90)$ arrangement having favorable properties like \pm 45 degree plies give buckling stability and carry shear, 0 degree plies give column stability and carry tension or compression, 90 degree plies carry transverse loads and reduce Poisson's effect. each of material is having its advantages and disadvantages properties and based on these properties tapered cone will show various values of stress and deformation. The effect of thickness and cone angle is also studied and results are discussed in the next part of paper.

The picture shown below is tapered cone which is used for analysis in this paper.

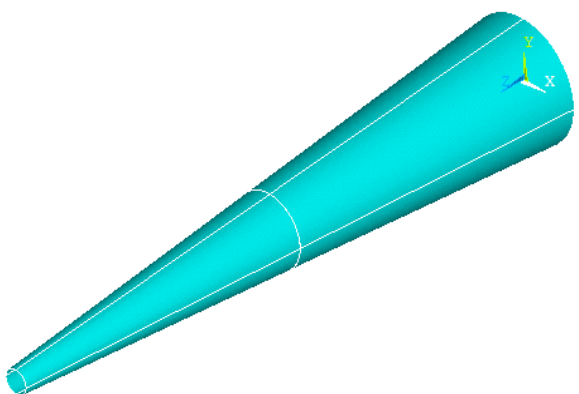

Fig:-Tapered composite cone

\section{Material and Their Properties:}

For the analysis three types of materials are used. In this article we took review of these material based on their properties

\subsection{Steel:-}

Steel is an alloy of iron and other elements, including carbon. When carbon is the primary alloying element, its content in the steel is between $0.002 \%$ and $2.1 \%$ by weight. The following elements are always present in steel: carbon, manganese, phosphorus, sulfur, silicon, and traces of oxygen, nitrogen and aluminum. Alloying elements intentionally added to modify the characteristics of steel include: manganese, nickel, chromium, molybdenum, boron, titanium, vanadium and niobium.

\subsection{Composites:-}

Typically made of a matrix and 1 or more reinforcements .They have different compositions, shapes and physical/chemical Properties. The type, distribution, size, shape, orientation, and arrangement of the reinforcement will determine the properties of the composites material and its anisotropy.

\subsubsection{Orthotropic Composite}

An orthotropic material has three planes of symmetry that coincide with the coordinate Planes. It can be shown that if two orthogonal planes of symmetry exist, there is always a third orthogonal plane of symmetry. Nine constants are required to describe this type of material. 


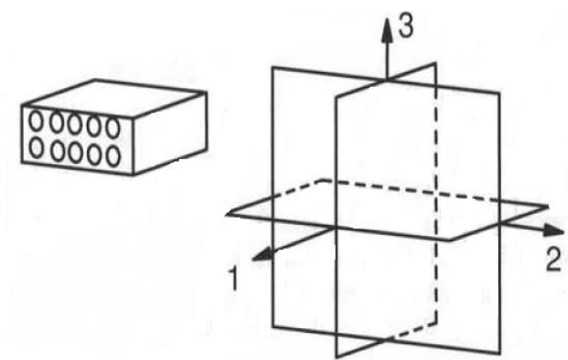

FIG: - Orthotropic material

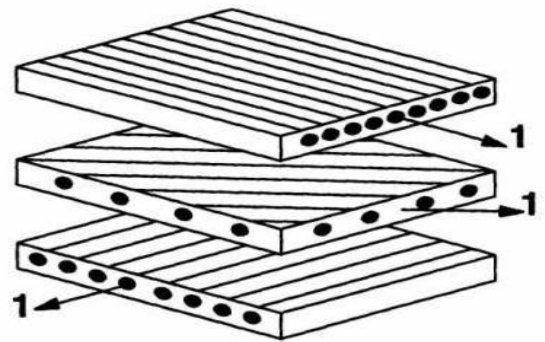

FIG: - laminated composite

The symmetry planes can be Cartesian, as depicted in Figure, or they may correspond to any other coordinate representation (cylindrical, spherical, etc.). For example, the trunk of a tree has cylindrical orthotropy because of the growth rings. However, most practical materials exhibit Cartesian orthotropy. A unidirectional fiber reinforced composite may be considered to be orthotropic. One plane of symmetry is perpendicular to the fiber direction, and the other two are orthogonal to the fiber direction and among themselves.

\subsubsection{Laminated Composite:}

A composite is constructed of layers, called lamina, that are bonded together. Each layer is composed of fibers and a polymer matrix material that is required to hold the fibers in position. The orientation angle of thefibers can change, which will, in effect, change the response of the tapered cone

The orientation angles of all of the layers are known a layup. For example, [0/45/90] is a composite layup with fibers in the top layer oriented at $0^{\circ}$, in the second layer at $45^{\circ}$, the third layer at $90^{\circ}$, fig. shows $[0 / 45 / 90]$ laminated composite.

\section{Geometry and boundary condition}

The cone simulated in this paper is a tapered cone with two cases. ANSYS software is used to simulate tapered cone .In Case 1 tapered cone is fixed at small end and free at the other end torsional load is applied at the free end of the cone as shown in figure 1. And case 2 tapered cone is fixed at big end and free at the other end .torsional load is applied at the free end of the cone as shown in figure 2

For the above two cases ,radius for small end is varied from $12 \mathrm{~mm}$ to $28 \mathrm{~mm}$ (in steps of $4 \mathrm{~mm}$ ) And big end radius is fixed as $100 \mathrm{~mm}$.for every five cases and for each material ,thickness of the cone is varied from $3 \mathrm{~mm}$ to $6 \mathrm{~mm}$.(for the laminated arrangement thickness is distributed equally in three layers).As is some cases during analysis tapered cone will show stress concentration ,to avoid this we are doing path operations and membrane stresses at middle section in the $\mathrm{z}$ direction are analyzed

Following figure shows tapered cone with boundary condition for case1 and case 2. In this paper we consider only case 1 for discussion.

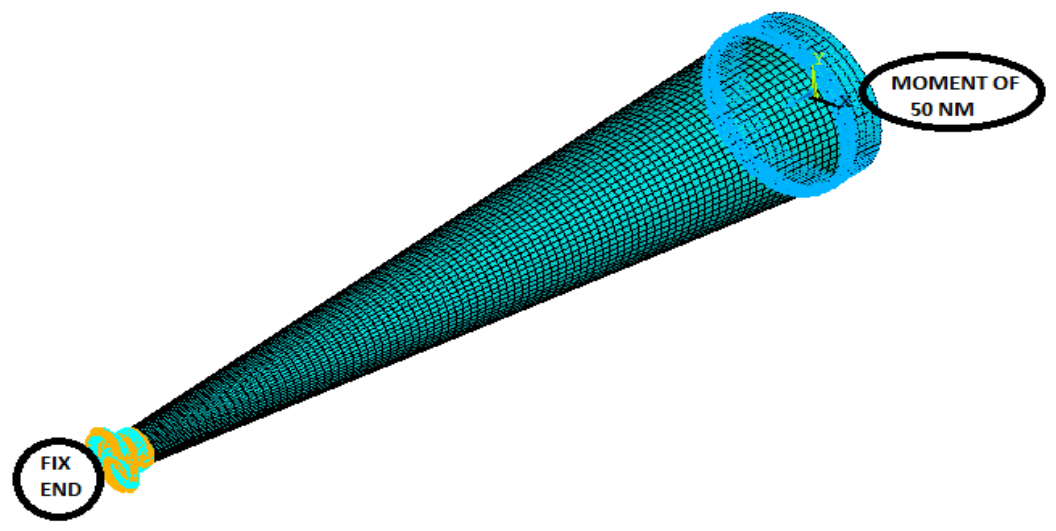

Fig:-Tapered cone with case 1 Boundary conditions 


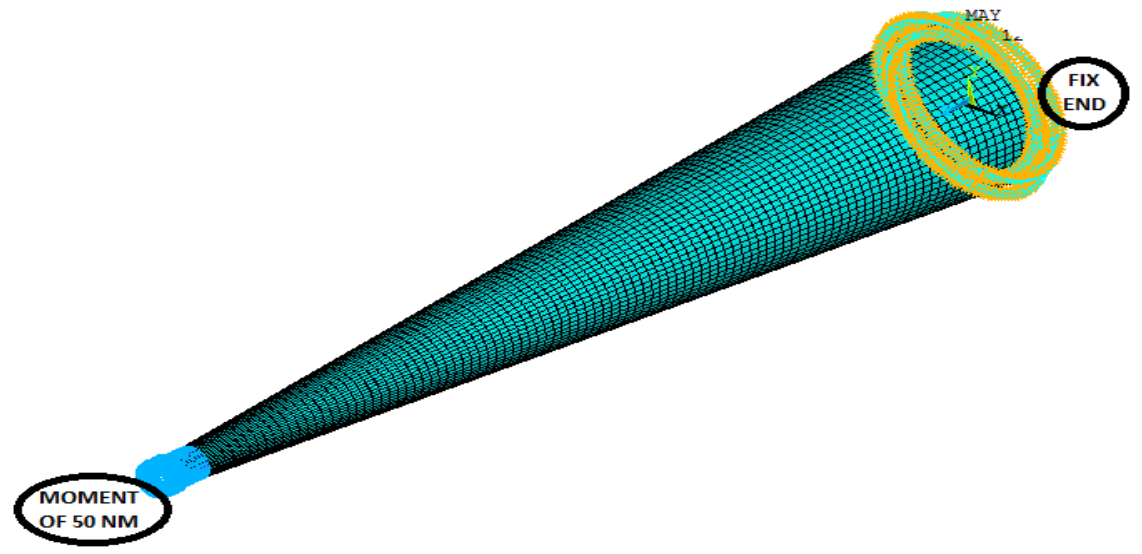

Fig:-Tapered cone with case 2 Boundary conditions

Material properties used throughout this study are shown in Table 1 and 2

For steel (Table 1)

\begin{tabular}{|l|l|l|l|}
\hline Ex (MPA) & $2.1 \mathrm{e} 5$ & Prxy & 0.3 \\
\hline
\end{tabular}

For orthotropic and laminated composite (UD AS4D/9310 Carbon/Epoxy) (Table 2)

\begin{tabular}{|c|c|c|c|c|c|}
\hline Ex (MPA) & $133.86 \mathrm{e} 3$ & Gxy(MPA) & $4.306 \mathrm{e} 3$ & Prxy & 0.301 \\
\hline Ey (MPA) & $7.706 \mathrm{e} 3$ & Gyz(MPA) & $2.76 \mathrm{e} 3$ & Pryz & 0.396 \\
\hline Ez (MPA) & $7.706 \mathrm{e} 3$ & Gxz(MPA) & $4.306 \mathrm{e} 3$ & Prxz & 0.301 \\
\hline
\end{tabular}

\section{Finite element modeling}

ANSYS was used to simulate the response of three different materials with different geometry and boundary conditions

For the steel and general orthotropic arrangement, element used is shell93 (8-Node Structural Shell) .Element type particularly well suited to model curved shells. For its midside node capability, the deformation shapes are quadratic in both in-plane directions. Shear deflection is included in this shell element (FSDT). The element has nonlinear and large strain capabilities.

For laminated composite arrangement element used is SHELL 91(8-Node Nonlinear Layered Structural Shell) .This element type may be used for layered applications of a structural shell model or for modeling thick laminates and sandwich structures (uses FSDT). Up to 100 different layers are permitted. The element has nonlinear and large strain capabilities.

- If applicable, SHELL99 is usually more efficient (smaller formulation time) than SHELL91. However, SHELL91 is more robust for nonlinear analysis.

Above two shell elements (SHELL93 AND SHELL 91) taken for analysis uses First order shear deformation theory (FSDT).

It is based on the following assumptions:

1. A straight line drawn through the thickness of the shell in the unreformed configuration may rotate but it will remain straight when the shell deforms. The angles it forms (if any) with the normal to the unreformed midsurface are denoted by $\emptyset \mathrm{x}$ and $\emptyset \mathrm{y}$ when measured in the $\mathrm{x}-\mathrm{z}$ and $\mathrm{y}-\mathrm{z}$ planes, respectively.

2. The thickness of the shell remains unchanged as the shell deforms.

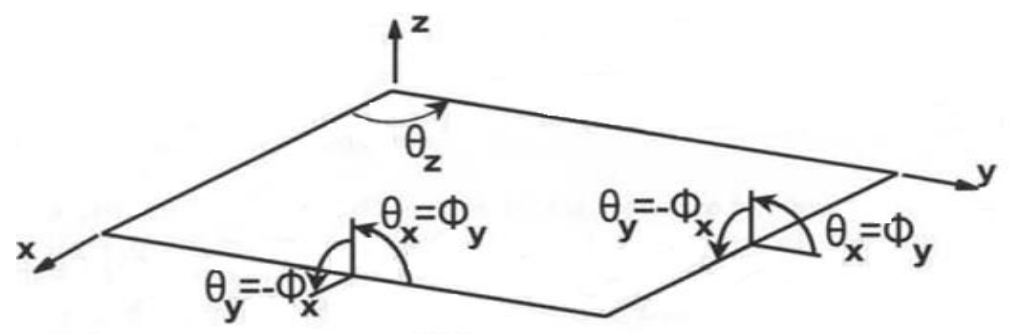

FIG. Convention for rotations of a plate or shell 


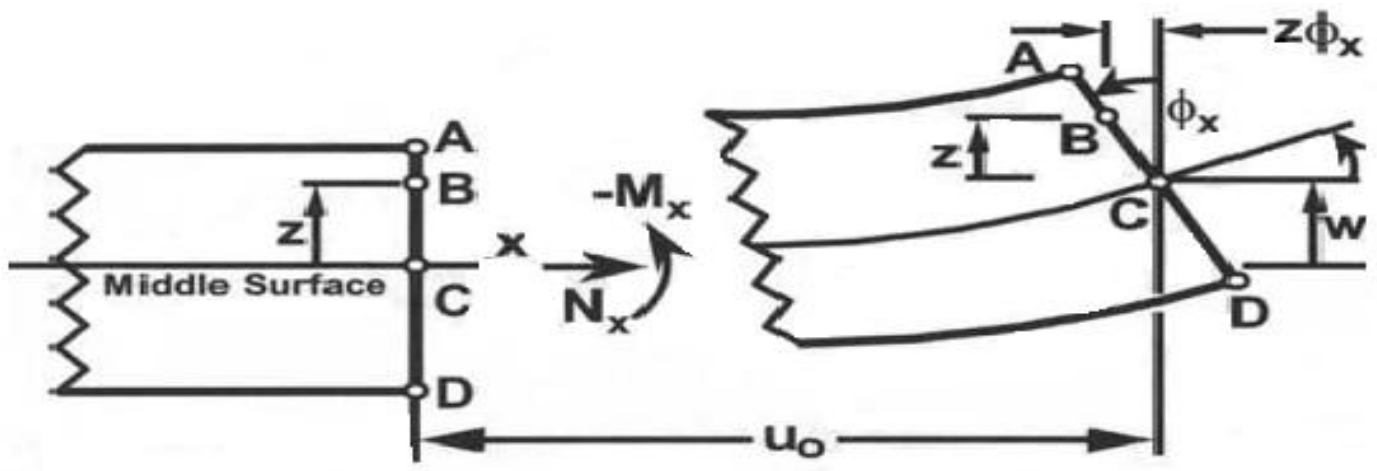

Unreformed Cross Section

Deformed Cross Section

FIG .Assumed deformation in FSDTI

\section{Results and Discussions:-}

Tapered cone with small end fixed and big end having moment $50 \mathrm{NM}$ is analyzed for each material and for every time we are increasing thickness of a cone from $3 \mathrm{~mm}$ to $6 \mathrm{~mm}$ (insteps of $0.3 \mathrm{~mm}$ ). Results are taken by varying small end diameter as 12, 16, 20, 24, 28 etc. This is for changing taper angle of cone because for various angles of cone deformation and stresses are changing and we have to consider these changes in angle for various applications of tapered cone such as automobile diffuser, tennis racket ,baseball shaft etc. Graphs are plotted as thickness vs. deformation as well as thickness vs. stresses for a cone.

First we are discussing effect of material properties, thickness and cone angle on deformation of a cone. As in structural analysis of different component, deformation plays very important role because it is a change in the shape or size of an object due to an applied force. So for given applied force if component is showing deformation within its limit values it will gives performance as per designer requirement. Following graphs plotted as thickness vs. deformation for three different materials.
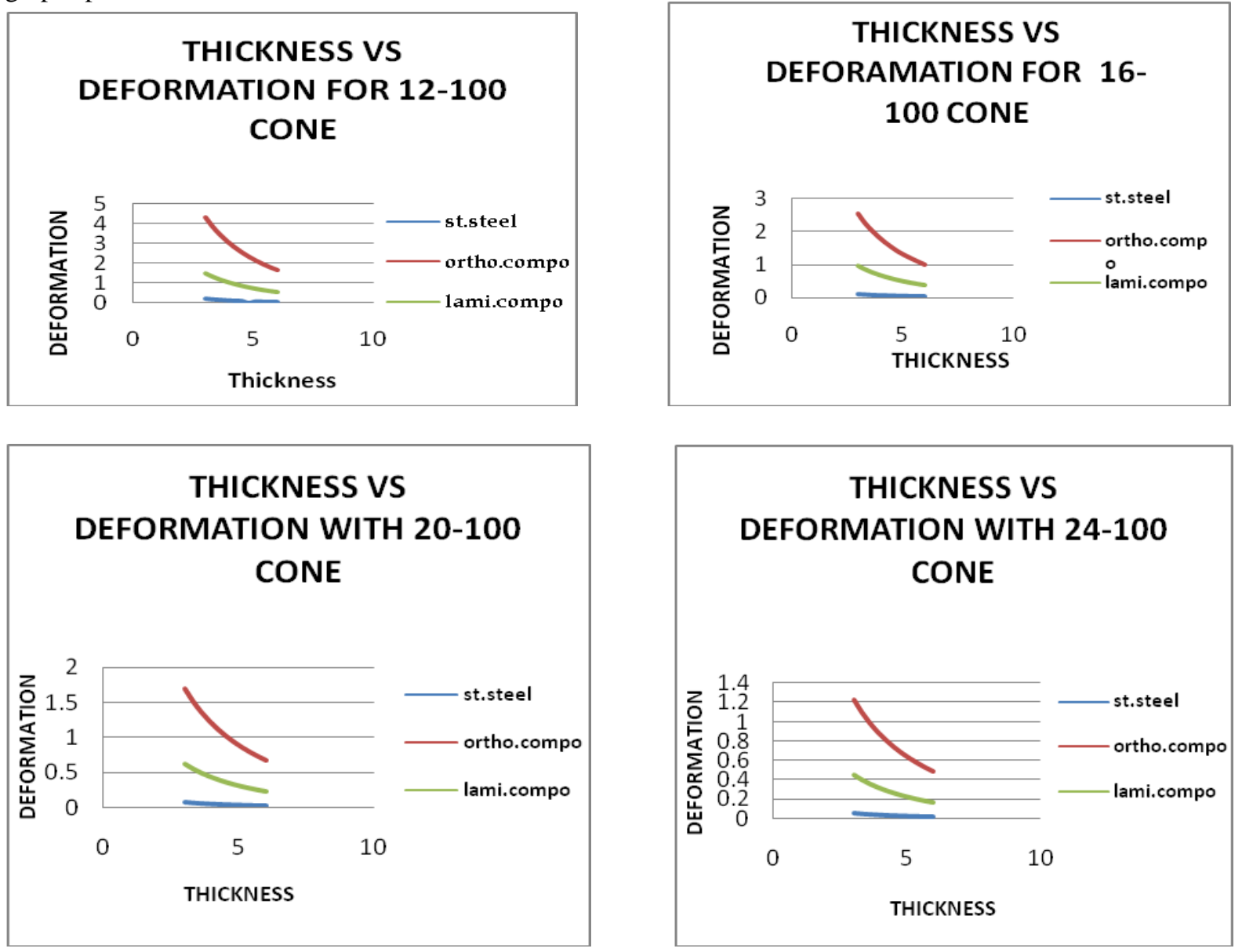


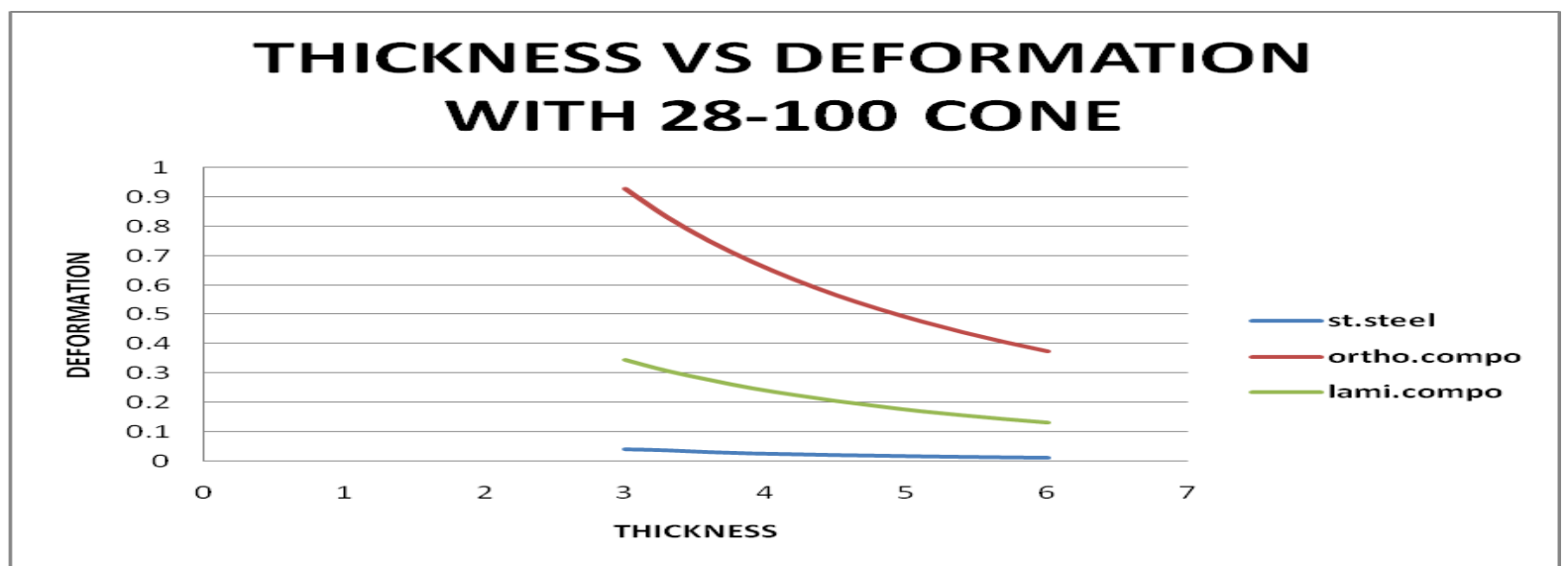

From above graphs it is seen that defromation for the tapered laminated composite cone is nearly same as for tapered steel cone but for tapered orthotropic composite cone it is more than remaining two materials.similarly as we are increasing thickness of cone deformation is gradually decreasing for each material .but rate of decrease of deformation is higher in case of orthotropic composite cone than other two material .As steel and laminated composite cone showing somewhat same values for defomation so laminated composite can be a best alternative in place steel cone where weight reduction has primary importance.

Along with above comparison based on values of deformation,pattern of deformation shown by steel and composite material is of very interasting for analysis engineer. Fig 7 nad 8 shows nature of deformation for steel and coposite material,it is seen that for in case of steel cone deforamtion pattern having bands of coloured ring but in composite cone it showing different band patterns.so while doing analysis with composite special attension sholuld be given on deformation pattern.

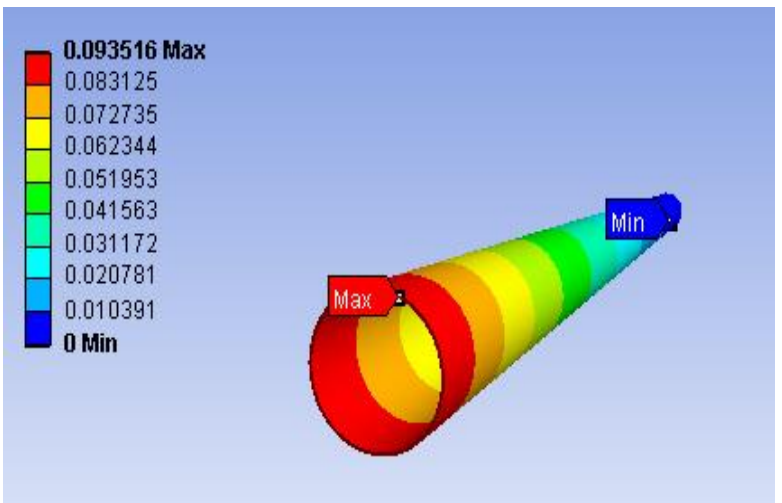

FIG. Deformation in steel cone

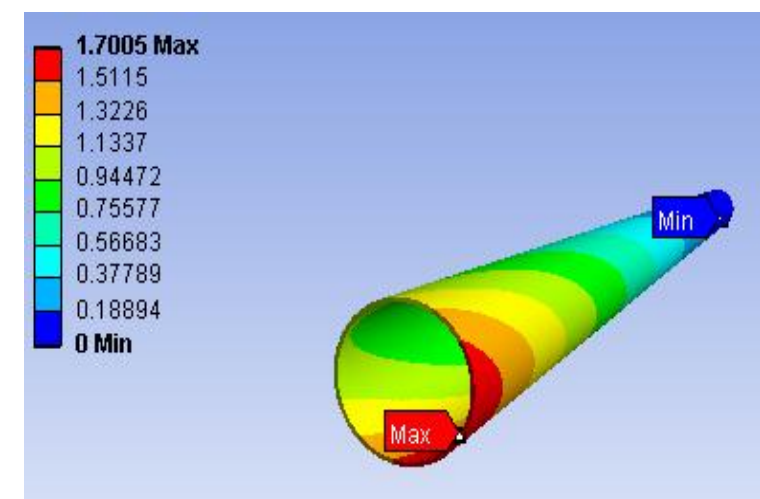

Fig .Deformation in composite cone

For above all the cases if we consider stresses in cone, it will show behavior as shown in following graphs. These graphs are plotted as thickness vs. stresses for three different materials.

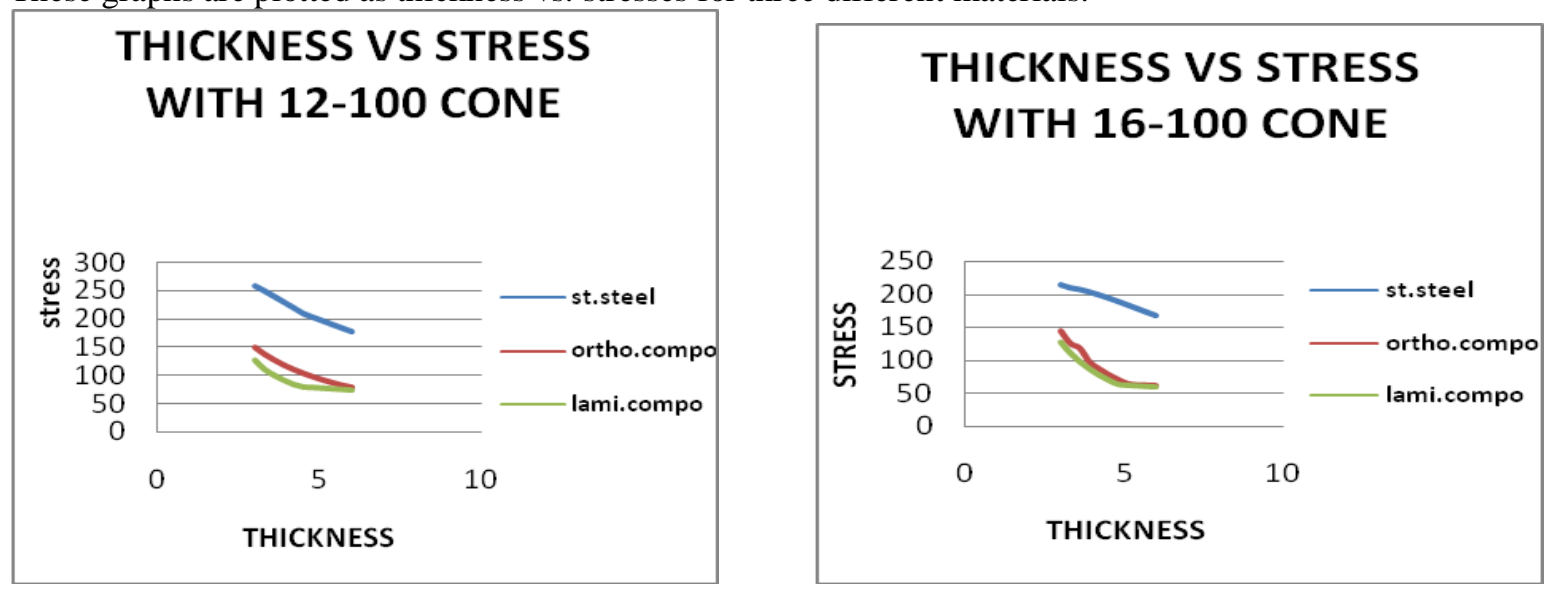



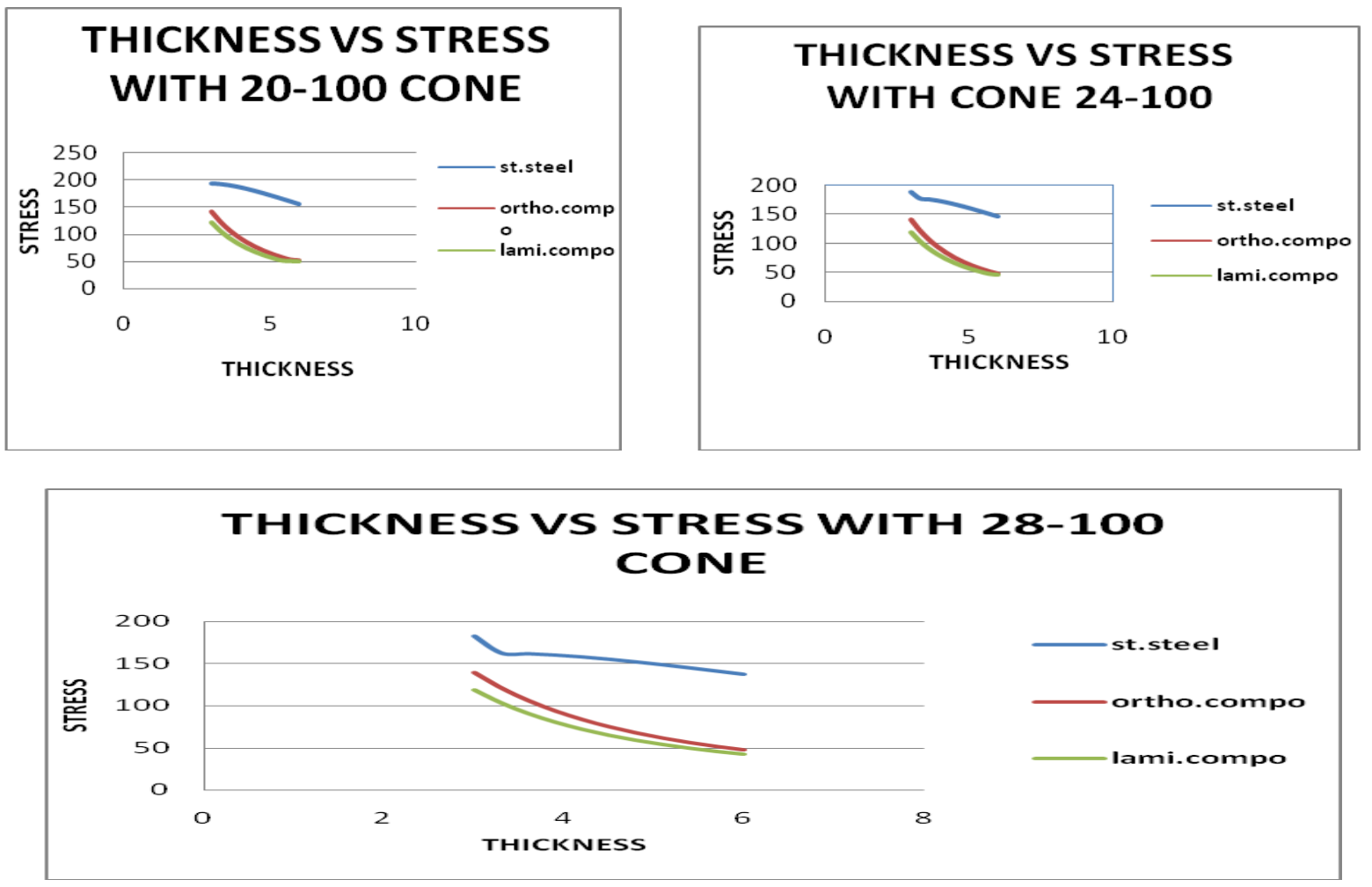

From above graphs of stresses vs. thickness for three different materials, we conclude that for steel tapered cone stresses developed are more as compared with other two material and out of three materials, laminated composite cone will show lowest von mises stress values. Similarly as we are increasing thickness of a cone, for each material stresses are decreasing as shown in graphs.

\section{Procedure for Membrane Stresses :-}

While analyzing tapered cone with boundary conditions explained above stresses get concentrated at free end of a cone .so it is interesting to calculate membrane stresses at centre of a cone .For cone with $20 \mathrm{~mm}$ small dia. And $100 \mathrm{~mm}$ large dia. and with case 1 membrane stresses are calculated for all three materials. Fig 5 is showing procedure for calculation of a membrane stresses. Path given for membrane stress calculation is starting from top most node of circle and moving in clockwise direction up to last node on a circle. Then some name is assigned to that path for our understanding. By using tool linear zed standard from ANSYS main menu membrane stresses are calculated for every thickness of a cone. it is observed that for every material as we increase thickness of a cone from $3 \mathrm{~mm}$ to $6 \mathrm{~mm}$ membrane stresses are decreasing .but membrane stresses in case of steel cone are more as compared with laminated composite cone .

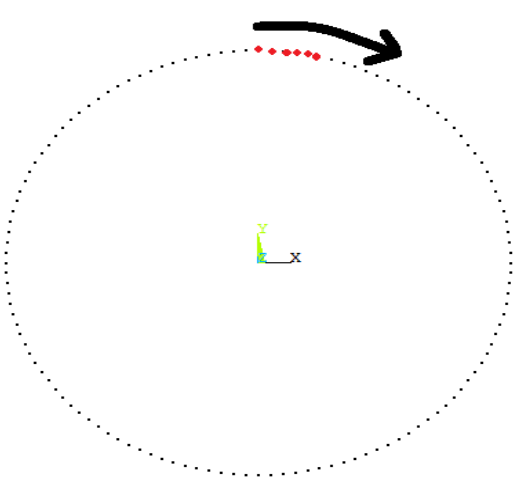

Fig5:-Membrane stress process

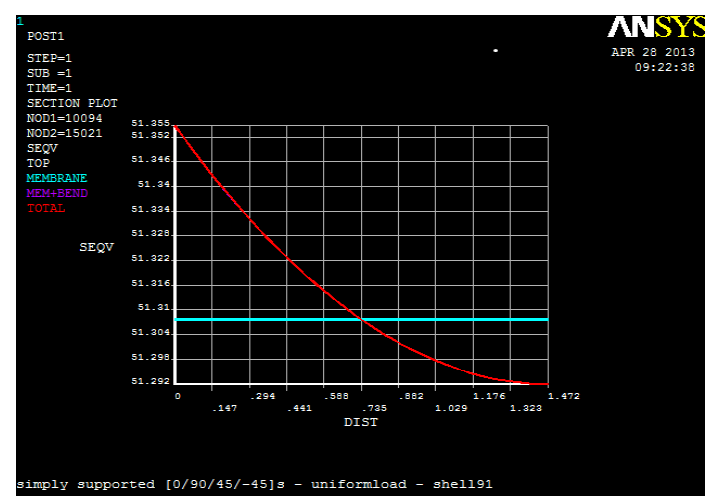

Fig:-membrane stress at middle of cone 


\section{Conclusion:-}

A number of analyses are performed in this design study, using a finite element model of the tapered cone for various cone angles, thickness of a cone and two boundary conditions. The model was developed using different structural shell elements in ANSYS12.0.

$>$ From the results it is observed that, in terms of stress composites have a better behavior than steel, and give lower values of stresses.

$>$ Also from stress point of view, laminated and orthotropic composites give similar levels of stresses.

$>$ However from a deformation point of view, laminated composites have a better behavior than orthotropic composites. This is because the interaction between the different lamina gives it additional stiffness.

Thus overall we can conclude that laminated composites are better than both orthotropic and isotropic (steel) materials.

\section{Theses:}

[1] Chethana Shankar Rao, "Analysis of tapered laminated composite tubes under tension and torsion". An Engg. Project Presented to the Faculty of the Graduate School of The University of Texas at Arlington. [May 2007]

[2] Brian Schmalberger, "Optimization of an Orthotropic Composite Beam". An Engineering Project Submitted to the Graduate Faculty of Rensselaer Polytechnic Institute Hartford, Connecticut. [May 2010]

\section{Journal Papers:}

[3] Choudhary S.S. and Tungikar V. B., “A simple finite element for nonlinear analysis of composite plates". International Journal of Engineering Science and Technology, Vol. 3, No.6, pp. 4897-4907. [2011]

[4] Reddy et al. / International Journal of Engineering, Science and Technology, Vol. 4, No. 2, 2012, pp. 177-190

\section{Books:}

[5] Ever J. Barbero, "Finite element analysis of composite materials", CRC press, New York.[2008]

[6] "Composites", ASM handbook, Volume 21, (2001) 